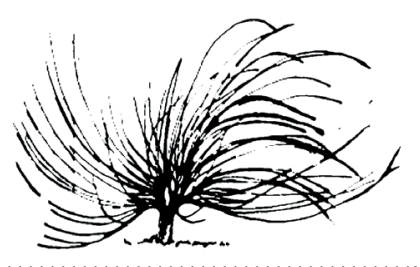

\title{
El currículum en el contexto costarricense: propuesta de definiciones para su conceptualización
}

César Toruño Arguedas ${ }^{1}$

Universidad Técnica Nacional

Costa Rica

ctoruno@utn.ac.cr

\begin{abstract}
Resumen
El presente ensayo realiza un análisis de la concepción del currículum en los enfoques curriculares técnico, práctico y crítico, como marco delimitador para la construcción de definiciones del currículum estructuradas a partir de la revisión teórica, una posición ecléctica de los enfoques práctico y crítico, la realidad del contexto costarricense y la premisa de utilizar definiciones para uso y práctica del currículum. Estas definiciones pretenden ser un insumo al análisis del currículum costarricense desde nuevas aristas de interpretación-acción y escenarios de transformación o reforma curricular.
\end{abstract}

Palabras clave: currículum, enfoques curriculares, concepto de currículum, Costa Rica.

\begin{abstract}
This essay makes an analysis of the conception of curriculum in the technical, practical and critical
\end{abstract}

Recibido: 17 de enero de 2019. Aprobado: 28 de octubre de 2019.

http://dx.doi.org/10.15359/rep.15-1.2

1 Doctor en Ciencias de la Educación y máster en Planificación Curricular. Ha sido asesor nacional en currículum, en el Consejo Nacional de Enseñanza Superior Universitaria Privada; consultor para el diseño y la evaluación de carreras en la educación superior; profesor universitario, y, actualmente, ejerce funciones en el Programa Éxito Académico de la Universidad Técnica Nacional. 
approaches as a framework delimiter for building structured curriculum definitions from a theoretical review, an eclectic point of view for the practical and critic approaches, the reality of the Costa Rican context, and the premise of using definitions for the use and practice of the curriculum. These definitions are intended as an input of analysis of the Costa Rican curriculum from new interpretation edges of actions and scenarios of transformation or curricular reform.

Keywords: curriculum, curricular approaches, curriculum concept, Costa Rica.

\section{Introducción}

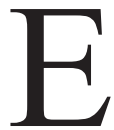
1 currículum ha sido una de las áreas pedagógicas con mayor diversidad de interpretaciones sobre su objeto de estudio y acción práctica, por lo que resulta acertada la aproximación conceptual elaborada por Guirtz y Plamidessi (1998, p. 6), según la cual el currículum es "un artificio vinculado con los procesos de selección, organización, distribución, transmisión y evaluación del contenido escolar que realizan los sistemas educativos". Sin embargo, a pesar de contener elementos básicos de lo que podríamos reconocer como currículum, las características y delimitaciones de este radican en los marcos conceptuales y reguladores propios de cada enfoque curricular, los cuales serán sintetizados, para efectos del presente ensayo, en tres categorías, a saber: técnica, práctica y crítica.

Posteriormente, se realizará una serie de delimitaciones conceptuales subyacentes en la construcción de las definiciones del currículum en Costa Rica. Ambos apartados permitirán una lectura contextual de las definiciones propuestas, las cuales serán esbozadas a partir del uso y la práctica del currículum en Costa Rica. Finalmente, se aportarán las conclusiones a las que ha permitido llegar la elaboración teórica del documento.

\section{El currículum: concepto, características y delimitaciones desde los enfoques técnico, práctico y crítico}

El currículum, como hecho cultural intencionado del ser humano para estructurar la transmisión-construcción de conocimientos, 
encuentra sus raíces en los terrenos de la historia antigua. En Occidente, ha sido constante la vinculación del surgimiento del currículum con el desarrollo de la educación en la Grecia Clásica. Aunque comprensible por el nacimiento de lo que hoy denominaríamos "centros educativos" o "instituciones educativas", resulta necesario ampliar, en futuras reflexiones, las otras regiones (Mesopotamia, América Precolombina, Asia y Egipto) que desarrollaron propuestas de acumulación-transmisión-creación de conocimientos, estructuradas a partir de un incipiente currículum, debate que trasciende a los objetivos del presente ensayo.

Posterior a la época antigua, el currículum occidental quedó reducido a las interpretaciones medievales de educación en el proyecto hegemónico religioso-imperial y los proyectos independientes de "benefactores" (nobleza y luego burguesía) con planes de acompañamiento y formación de artistas, escritores, filósofos y otros. Tanto en la Época Antigua como en la Edad Medieval, no existieron necesidades de masificación de la educación y ampliación de la cobertura, por lo que esta quedó reducida para un muy selecto grupo de individuos. El proceso tendría una ruptura en la Época Moderna (marcada por el Renacimiento y la Revolución Francesa), por la expectativa de mejora de los individuos y los pueblos, mediante una "transmisión" de la cultura universal y formación de los "nuevos" ciudadanos.

No obstante lo anterior, la historia del currículum occidental encuentra un punto de inflexión en el siglo XIX, por cuanto las demandas de la Ilustración y la Revolución Francesa, el desarrollo y la consolidación de los Estados Nacionales en Europa y América (excolonias europeas) y la consolidación de la Revolución Industrial implicaron un aumento de escolarización, a través de un incremento de la cobertura (matrícula masiva de estudiantes para primeros años, con el afán de enseñarles habilidades comunicativas en su lengua materna, dominio de principios básicos de las matemáticas, formación de principios para la vida en los nacientes Estados Nacionales y dogmas fundamentales de la fe cristiana) y una consolidación de la educación tanto secundaria como universitaria, con tasas de cobertura dirigidas a un pequeño porcentaje de la población.

En nivel global, el fin del siglo XIX e inicio del XX implicó la consolidación del capitalismo como fuerza rectora del orden económico-político en Occidente; el surgimiento de movimientos de resistencia y la lucha contra las desigualdades propulsadas por el modelo económico; 
la batalla expansionista y neocolonial de las potencias europeas para dominio de mercados; el conflicto militar entre las potencias (Primera y Segunda Guerra Mundial), y el surgimiento de Estados Unidos como potencia militar, económica, política y cultural (campañas comerciales, producción de ropa, comidas enlatadas y cine).

La sociedad estadounidense, posicionada como potencia industrial y militar ascendente, enfrentó el desafío de producción, innovación y legitimidad, mediante la construcción de un complejo sistema educativo (fraccionado por comunidades, Estados y lineamientos federales) que respondiera a las demandas de producción (mano de obra especializada), militares (disciplina y conocimientos básicos), académicas (mejora de la formación), políticas (formación de ciudadanos) y sociales (inclusión de las mujeres). En este contexto, surge el currículum como un área que permitiría mejorar la planificación y producción del sistema educativo, al conceptualizarse como una extrapolación de los principios de organización, control regulación y evaluación de las empresas.

Las anteriores circunstancias determinan el surgimiento del enfoque técnico o pedagogía por objetivos (Gimeno, 1997); es decir, el enfoque del diseño, desarrollo y evaluación del currículum, caracterizado por extrapolar principios empresariales a la organización administrativa y vivencia pedagógica de los centros educativos, en tanto

considera la sociedad y la cultura como una 'trama' externa a la escolarización y al currículum, como un contexto caracterizado por las 'necesidades' y los objetivos sociales deseados a los que la educación debe responder, descubriendo esas necesidades y desarrollando programas con el fin de alcanzar los propósitos y objetivos de la sociedad (Kemmis, 1998, p. 112).

A finales de la década de 1940, Tyler (1973) presenta el libro Principios básicos del currículum, el cual se convierte en el referente del enfoque técnico, al proponer una organización del currículum a partir de objetivos que determinarán la mediación y evaluación. En su propuesta, Tyler (1973) establece los fundamentos del currículum (psicológicos, sociológicos y disciplinares) que determinan la selección de contenidos, la cual, a su vez, es producto de consideraciones filosóficas (ideal de ser humano) y la psicología del aprendizaje, para dar paso a 
una planificación curricular orientada al cumplimiento de objetivos de aprendizaje desde un paradigma conductista.

Aunque revolucionaria en su momento (por implicar la estructuración y sistematización de la planificación curricular), la propuesta de Tyler enfrentaba vacíos conceptuales y pragmáticos que fueron abordados, en las décadas de 1960 y 1970, por diversos autores, siendo referentes Gagné y Taba. En el caso de Gagné, su proposición curricular asume el principio de que se debe elegir "una de las cinco clases de su taxonomía, se establecieran tanto las condiciones internas que debe poseer el sujeto para poder desarrollar esa capacidad (alcanzar el nuevo objetivo) como las condiciones externas o medios con que se lograrán" (Clemente, 2010, p. 274), por lo cual representa un avance con respecto a la sistematización de objetivos (taxonomías) y en la implementación por parte de los docentes.

Paralelamente, Taba (1974) desarrolla una propuesta curricular continuadora de los elementos de Tyler, pero ajustada a una sociedad en plena Revolución Científica-Tecnológica, determinada por la Guerra Fría, la fractura social en Occidente (movimientos sociales, brecha generacional y cuestionamiento a la institucionalidad) y la expansión económica post-Segunda Guerra Mundial. Al respecto, la autora reconoce que la presión a "favor de la revisión del currículo proviene de los cambios drásticos en la tecnología y la cultura, que van desde la mecanización hasta la energía atómica, las demandas insaciables de la industria en expansión de la mano de obra inteligente" (Taba, 1974, p. 15).

La propuesta de Taba (1974) reitera que la planificación curricular es una toma de decisiones sustentadas en el estudiante, el proceso de aprendizaje, exigencias culturales y el contenido de las disciplinas, pero estructurado y sistematizado a partir de las siguientes etapas: a) diagnóstico de las necesidades; b) formulación de objetivos; c) selección del contenido y organización de este; d) selección de las actividades de aprendizaje; e) organización de las actividades de aprendizaje, y f) determinación de lo que va a evaluar, así como de las maneras y medios para medirlo.

A pesar de las mejoras propuestas por Gagné y Taba, el enfoque técnico mantuvo un sesgo de control, previsión y evaluación que, como cuestiona Gimeno (1997, p. 10), aspira a

lograr diseños o programaciones muy estructuradas del proceso de enseñanza-aprendizaje, la intención de lograr el que cada uno 
de esos diseños se ajuste a objetivos específicos, la huida de la ambigüedad, de los objetivos no formulados en esos términos, la obsesión por el cómo formularlos, la búsqueda de la eficacia mensurable de los tratamientos pedagógicos que persiguen esos diseños ajustados.

Desde la propuesta técnica, el currículum es un "un plan para el aprendizaje; por consiguiente, todo lo que se conozca sobre el proceso del aprendizaje y el desarrollo del individuo tiene aplicación al elaborarlo" (Taba, 1974, p. 25). En la mayoría de los casos,

parte de una determinación de metas y objetivos específicos, lo que indica algún tipo de selección y organización del contenido, y destaca ciertos modelos de aprendizaje y enseñanza, sea por exigencia de los objetivos o de la organización del contenido. Finalmente, incluye un programa de evaluación de los resultados (Taba, 1974, p. 24).

La década de 1960 vertería en el debate educativo los cuestionamientos provenientes desde las ciencias sociales y sociedades occidentales marcadas por movimientos sociales, políticos y culturales, así como por el ascenso y la rapidez de la Revolución Tecnológica. Las críticas al currículum se fundamentaban en las premisas de que, por una parte, respondía a los intereses capitalistas, al proyecto hegemónico (posición crítica) y a que tanto su estructura de diseño como de operacionalización era ineficaz e irrelevante en sociedades tan complejas; por otra, al cuestionamiento por "la burocratización, la desideologización y la desconexión con la práctica" (Clemente, 2010, p. 280).

En el caso de las críticas al diseño y a la operacionalización, se constituye una alternativa bajo lo que será denominado enfoque práctico, en el que se debe considerar la sociedad y la cultura "como un tipo de sustrato, pero adopta un punto de vista más activo acerca del papel de la educación, de las escuelas y de los profesores, en su contribución a la sociedad y a la cultura" (Kemmis, 1998, p. 112).

Dicho enfoque (práctico) encuentra a Schwab y Stenhouse como referentes; sin embargo, la propuesta de Stenhouse consolida la ruptura con el enfoque técnico por cuanto el problema central de estudio del currículum es "el hiato existente entre nuestras ideas y aspiraciones, y 
nuestras tentativas para hacerlas operativas" (Stenhouse, 2003, p. 27), aceptando y promoviendo la variabilidad de opciones, situaciones y condiciones que determinan el currículum y que, por tanto, hacen imposible el control. En este sentido, se define el currículum como "una tentativa para comunicar los principios y rasgos esenciales de un propósito educativo, de forma tal que permanezca abierto a discusión crítica y pueda ser trasladado efectivamente a la práctica" (Stenhouse, 2003, p. 29), en el marco de un modelo de proceso en el cual las evaluaciones son flexibles para responder al contexto.

A partir de esta nueva visión del currículum, Stenhouse (2003) establece el análisis y la planificación curricular, con base en una visión de proyecto regida por principios para la selección del contenido, desarrollo de una estrategia de enseñanza, adopción de decisiones relativas a la secuencia o diagnóstico de los estudiantes. Asimismo, Stenhouse se fundamenta en un estudio empírico y evaluación del progreso de alumnos; el estudio y la evaluación del progreso de los profesores; orientación para implementar el currículum en diversos contextos, e información sobre la variabilidad de efectos en diferentes contextos. Por último, como tercer eje de análisis, se establece la justificación, es decir, una formulación de la intención o finalidad del currículum, susceptible al análisis crítico.

El proyecto curricular de Stenhouse (2003) se caracteriza como un proceso no centrado en los objetivos (cualidad del enfoque técnico), evaluado con técnicas e instrumentos más allá del reduccionismo de la prueba del conductismo, el análisis (por parte de teóricos, investigadores y docentes) del potencial del currículum, la condicionalidad entendida como la relación del potencial del currículum con las condiciones contextuales del centro educativo y la elucidación. Todo esto implica los aportes del currículum implementado al esclarecimiento de los problemas planteados por el cambio en el sistema educativo, advirtiendo el papel del docente y su calidad para la ejecución del diseño curricular.

Los enfoques técnicos y prácticos recibieron cuestionamientos desde la pedagogía crítica. En el primero de los casos, por la alineación del currículum al proyecto hegemónico y la extrapolación de principios empresariales que deformaban el proceso de construcción de aprendizajes y formación del ser humano; en el enfoque práctico, por omitir la influencia del currículum oculto y el proyecto hegemónico, 
manteniendo la premisa del currículum aislado de grupos de presión, intereses y redes de poder, motivo por el cual se constituye el enfoque crítico, sociocrítico o revolucionario.

El enfoque crítico surge en las décadas de 1950-1960 y es producto de investigaciones educativas elaboradas a partir de los aportes de la teoría crítica de la Escuela de Fráncfort, estudios estructuralistas marxistas y neomarxistas, basados en la premisa de que el sistema educativo tiene una interacción dialéctica con el contexto, incluyendo ideologías, grupos de presión, redes de poder, proyectos hegemónicos, mecanismos de resistencia y liberación, entre otros. Kemmis (1998, p. 113) sintetiza este enfoque al afirmar que

parte de la premisa de que las estructuras sociales no son tan racionales y justas como generalmente se piensa. Por el contrario, afirman, las estructuras sociales están creadas mediante procesos y prácticas distorsionados por la irracionalidad, la injusticia y la coerción, y tales distorsiones han calado muy hondo en nuestras interpretaciones del mundo.

De la premisa anterior, se desprende que el currículum no es neutro sino "un territorio controvertido y hasta conflictivo, respecto del cual se toman decisiones, se siguen opciones y se actúa por orientaciones que no son las únicas posibles" (Gimeno, 2010a, p. 29), respondiendo a la estructura de un currículum oculto (Torres, 1998). Se puede afirmar que, el currículum "es un campo de batalla que refleja otras luchas: corporativas, políticas, económicas, religiosas, identitarias, culturales" (Gimeno, 2010a, p. 37).

En línea con lo expuesto, el enfoque crítico reivindica el currículum como un hecho cultural, una "una opción históricamente configurada que se ha sedimentado dentro de un determinado entramado cultural, político, social y escolar; está cargado, por lo tanto de valores y supuestos que es preciso descifrar" (Gimeno, 2001, p. 18). Igualmente, implica un "proyecto selectivo de cultura, cultural, social, política y administrativamente condicionado, que rellena la actividad escolar, y que se hace realidad dentro de las condiciones de la escuela tal como se halla configurada" (Gimeno, 2001, p. 40). También, se busca una difusión de los códigos culturales (Gimeno, 2010a), la incorporación tanto de contenidos como de metodologías innovadores dirigidos a la 
liberación, para evitar que los currículum sean "pobres, repetitivos, sin sabores" (González, 2010, p. 174), sin obviar una visión globalizadora del proyecto educativo, es decir, la inclusión (al centro educativo y al acto educativo) de las "necesidades vitales de los individuos para desenvolverse en sociedad, destrezas y habilidades consideradas fundamentales" (Gimeno, 2001, p. 65).

Adicionalmente, el enfoque crítico exige un compromiso con la justicia curricular, lo cual significa la "inclusión, representación, aportaciones y valoraciones de las personas, colectivos, grupos y culturas que están presentes en las aulas y en la sociedad más amplia en la que está ubicado el centro escolar" (Torres, 2010, p. 100), que permita la legitimidad del proceso y la equidad curricular, o sea "la distribución del currículum común-básico de manera 'justa', igualitaria a la población escolar, independiente de su procedencia social, económica, étnica, geográfica o religiosa, etc.” (Magendzo, 2008, p. 96). Esto se vincula con el acceso de diferentes actores al proceso curricular y al conocimiento, habilidades, sensibilidades, destrezas y actitudes adquiridas.

La exposición de los enfoques técnico, práctico y crítico realizada en el presente apartado permite establecer la comprensión del currículum como concepto asociado a circunstancias del proceso educativo (macro- y mesoplanificación), institucional (centros educativos); al acto educativo (contextualización curricular en el aula) y a las subjetividades de los participantes; al contexto profesional (enfoques), sociocultural, económico y político; a elementos y factores determinantes para la construcción de los diferentes conceptos, algunos de los cuales serán abordados a continuación.

\section{Concepción del currículum en el contexto costarricense}

A diferencia de la evolución del concepto de currículo durante la segunda mitad del siglo XX, la construcción teórica en Costa Rica encuentra su impulso durante la década de 1990, vinculado a los esfuerzos nacionales para la mejora de los procesos de aprendizaje. Se encuentra como referente clásico la propuesta de Bolaños y Molina (1992), según la cual

El currículo está constituido por las experiencias de aprendizaje que vive el alumno, dentro o fuera del ámbito escolar, bajo la orientación o motivación del docente. Estas experiencias 
pretenden alcanzar los fines y objetivos de la educación y pueden ser programadas o emerger durante el desarrollo cotidiano del proceso educativo, debido a la constante interrelación escuela-comunidad (Bolaños y Molina, 2007, p. 24).

A finales de la década de 1990, el currículo y la planificación curricular es un proceso de "toma de decisiones, en cuanto a metas, objetivos y procesos de enseñanza y aprendizaje que se propone alcanzar la institución educativa. La toma de decisiones involucra a los interesados en la formación académica, personal y laboral del profesional" (Mora y Herrera, 1998, p. 12). Por su parte, Garbanzo (2001, p. 27) definió el currículo como "un medio orientador del aprendizaje, el cual se orienta por diferentes posiciones filosóficas, políticas, ideológicas y paradigmáticas, el cual busca la concretización de la política educativa según los fines y objetivos de la educación" (Garbanzo, 2001, p. 27).

Por tanto, se conceptualiza el currículo más allá del proceso de aprendizaje, en línea con la evolución internacional del enfoque práctico y crítico; así mismo, se integran especificaciones técnicas sobre las áreas y ejes curriculares. Al respecto, Mora (2001, p. 151) define las áreas curriculares a partir de que "tienen una función integrado$\mathrm{ra}$, que permite la relación vertical y horizontal entre los contenidos curriculares" y los ejes como "las grandes ideas que traducen la intencionalidad del proceso educativo tanto implícito como explícito en el plan de estudios. Los ejes orientan el desarrollo de las competencias de los estudiantes integrando la formación académica con la formación personal (Mora, 2001, p. 151).

En la actualidad, las reflexiones curriculares en Costa Rica coinciden en que "El currículo es un proceso social, por lo que en su elaboración intervienen una gran cantidad de elementos que se deben tomar en cuenta" (Jiménez, 2008, p. 63) y en que se "abarca mucho más que el contenido del plan de estudios, pues se relaciona con la forma en que el mismo se organiza y estructura para facilitar el aprendizaje" (Rodríguez, 2013, p. 121).

Lo anterior, comprendiendo que el currículo

se encarga del diseño de actividades de aprendizaje secuenciadas con el fin de formar-educar personas en función de un determinado perfil de egreso, con una serie de características y 
conocimientos, llamado comúnmente, perfil de salida, y cuya definición es producto del análisis sociohistórico, epistémico y pedagógico de la profesión que se enseña (González, 2018, p. 10).

Con base en la anterior revisión teórica internacional y nacional, resulta necesario realizar una propuesta de definición curricular pertinente y relevante para el contexto costarricense.

\section{Algunas delimitaciones de la definición de currículum}

El recuento de las diversas concepciones del currículum resulta necesario para establecer un acercamiento conceptual con términos complementarios a los mencionados en el apartado anterior y requerido para la comprensión de la conceptualización por esbozar en la siguiente sección.

Definir el currículum es, o al menos debería ser, el primer paso del diseño curricular comprendido como el "proceso de planificación, darle forma y adecuarlo a las peculiaridades de los niveles escolares" (Gimeno, 2001, p. 224) y como un "boceto, esquema, plano, etc.; es decir, a una representación de ideas, acciones, objetos, de modo tal que dicha representación opere como guía orientadora a la hora de llevar el proyecto curricular a la práctica" (Casarini, 2005, p. 113). Puede ser establecido por cualquier agente del sistema educativo (incluidos los estudiantes y familias, si, en un sistema educativo democratizado, progresista e innovador, formaran parte activa de la planificación del proceso de aprendizaje).

Tradicionalmente, ha existido una asociación tácita entre el concepto de currículum, el programa de estudio y su aplicación en el aula. De esta visión, resulta referente la definición de Escudero et al. (2000, p. 28), según la cual, el currículum "se refiere a todo el ámbito de experiencias, de fenómenos educativos y de problemas prácticos, donde el profesorado ejerce su oficio y el alumnado vive su experiencia escolar". Esta asociación ha construido, explícita e implícitamente, una ambigua conceptualización de lo curricular, que invisibiliza el proceso de construcción, escenarios de transformación, impactos e implicaciones y, no menos importante, cercena las posibilidades de acción-reflexión sobre ello.

El currículum es mucho más que el programa de estudio, es "el conjunto de prescripciones oficiales respecto a la enseñanza emanadas del Poder central [que] recoge lo que en cada momento cultural y social es definido como los conocimientos, habilidades, valores y experiencias 
comunes y compartidas por un pueblo" (Zabalza, 2004, p. 15). Por tanto, el currículum se concibe como un espacio de transmisión del conocimiento oficial (Apple, 1996a), construido en el marco de políticas culturales, políticas y económicas que inciden en el sistema educativo (Apple, 1996b), las cuales responden a redes de poder públicas y privadas (Apple, 1997) y establecen un proyecto selectivo de cultura (Gimeno, 1998 a) en el que es posible construir la emancipación o espacios de resistencia (Kemmis, 1998, 1997, 1984).

Por lo anterior, resulta necesario establecer la diferencia entre el currículum explícito y el oculto, los cuales, en términos de Torres (1998, p. 198), se definen de la siguiente manera:

El currículum explícito u oficial aparece claramente reflejado en las intenciones que, de una manera directa, indican tanto las normas legales, los contenidos mínimos obligatorios o los programas oficiales, como los proyectos educativos de centro y el currículum que cada docente desarrolla en el aula. El currículum oculto hace referencia a todos aquellos conocimientos, destrezas, actitudes y valores que se adquieren mediante la participación en procesos de enseñanza y aprendizaje, y en general, en todas las interacciones que se suceden día a día en las aulas y centros de enseñanzas.

Como podrá apreciarse en las definiciones construidas en el siguiente apartado, la división entre currículum explícito y oculto es implícita en concepciones que pretenden ser pertinentes, a partir del uso y de la práctica del currículum (coherente con la separación contexto de formulación y contexto de realización que sugiere Lundgren - 1992-), pero que, bajo ninguna circunstancia, renuncian a la visión crítica de ambos escenarios (explícito y oculto) ni a los códigos curriculares, es decir, el "conjunto de principios según los cuales forman la selección, la organización y los métodos de transmisión" (Lundgren, 1992, p. 21).

Las definiciones responden a la premisa de que "Pensar en el currículum es pensar en cómo actúa e interactúa un grupo de personas en ciertas situaciones. No es describir y analizar un elemento que existe aparte de la interacción humana" (Grundy, 1998, p. 21); por tanto, deben ser analizadas como concepciones generadoras de la reflexión y acción curricular y no como limitantes de un currículum bonsái, es 
decir, un currículum diseñado, desarrollado e implementado para restringir el desenvolvimiento de los agentes educativos.

Lo anterior, a pesar de que, en primer lugar, el currículum costarricense preuniversitario y universitario se ha caracterizado por la centralización curricular, o sea la estructura educativa en la que "la autoridad que elabora el currículum oficial-nacional-básico-común está ubicada en el organismo central de los ministerios o secretarías ministeriales de Educación, que poseen los recursos humanos, financieros y tecnológicos para tomar decisiones sobre políticas públicas respecto al currículum" (Magendzo, 2008, p. 68).

En segundo lugar, otra limitante de la propuesta de conceptualización esbozada en este ensayo radica en que, tradicionalmente, se ha caracterizado al currículum como una estructura de planificación y control del proceso educativo, integrada por diversas acciones pedagógicas y administrativas. Estas últimas van dirigidas a la consecución de aprendizajes delimitados por objetivos de ciclos, nivel y asignatura, construidos a partir de fundamentos curriculares (psicológicos, sociales, pedagógicos, filosóficos y otros) operacionalizados en una selección y organización de los contenidos; a mecanismos de medición o evaluación, en tanto, la consecución de objetivos sería observable y medible mediante diferentes pruebas (nacionales y aquellas elaboradas por cada docente) a lo largo de la vida escolar; y a procedimientos de control social y ético (nota de conducta).

Los conceptos de currículum presentados a continuación asumen implícitamente que, con respecto a las funciones de la educación y paradigmas, el currículum "a veces las refleja explícitamente, pero también están en las condiciones dentro de las que éste se desarrolla" (Gimeno, 1998, p. 107) y que es "la expresión del conflicto, y por la toma de conciencia de que el currículum constituye uno de los principales agentes en la introducción, preparación y legitimación de las formas de vida social" (McLaren, 1997, p. 55). Esto, sin obviar la reivindicación del docente como intelectual transformador (Giroux, 1997), en cuanto "el profesorado hace políticas desde abajo o, lo que es lo mismo, rompe la línea política impuesta desde arriba, aunque dentro de unos límites" (Gimeno, 2010b, p. 225).

Por último, resulta necesario indicar que la propuesta de conceptualizaciones del currículum amplía los niveles de la planificación curricular indicados por Gimeno (2010a): a) proyecto educativo (el 
texto curricular), b) currículum interpretado por el profesorado y los materiales, c) currículum realizado en prácticas, d) efectos educativos reales (plano subjetivo de los estudiantes) y e) efectos comprobables y comprobados.

\section{Currículum en Costa Rica: algunas definiciones posibles}

En los segmentos previos, se indicaron las características y concepciones básicas del currículum, según los enfoques técnico, práctico y crítico, así como delimitaciones adicionales que resultaban necesarias para la comprensión de la propuesta de concepciones curriculares por esbozar en el presente apartado.

La estructura de la propuesta asume la premisa de que no puede existir una única definición de currículum, pues este debe ser comprendido a partir de su uso y práctica, motivo por el cual se sugiere la definición del currículum vinculada al área del conocimiento, como referente generalista. Esta permitirá construir la definición del currículum como proceso, la que a su vez es el marco delimitador de las acepciones del currículum como producto, currículum procesado, currículum como práctica educativa, currículum como vivencia educativa y currículum inesperado.

Las concepciones se construyen basadas en una posición ecléctica de los enfoques prácticos y críticos, con el horizonte de hacer posible su interpretación, análisis y práctica en la realidad del sistema educativo costarricense y con el supuesto de ser utilizados para el currículum construido, administrado y ejecutado por el Ministerio de Educación Pública, las universidades tanto públicas como privadas y por proyectos particulares de educación formal.

\subsection{Currículum como área del conocimiento}

El currículum es un área pedagógica que investiga, analiza y sistematiza el conocimiento sobre diseño, operacionalización, evaluación y mejora de los procesos educativos dirigidos a la transmisión, reproducción, transformación y construcción de saberes, habilidades, destrezas, sensibilidades y actitudes en el marco de la educación formal.

\subsection{Currículum como proceso}

El currículum es el proceso de interacción dialéctica entre un proyecto cultural, económico, político y social, que tiene como 
objetivo planificación, control, operacionalización y evaluación de los procedimientos educativos dirigidos a la transmisión, transformación y construcción de conocimientos, habilidades, destrezas, sensibilidades y actitudes a aprender y desaprender, por parte del estudiante. Para ello, se estructura una planificación administrativa-pedagógica y financiera, así como una organización del poder, espacio y tiempo educativo, junto a la distribución de conocimientos por áreas, asignaturas o especialidades, el diseño de un programa de estudio (producto tangible de la selección y orden de contenidos), mediaciones didácticas, actividades vivenciales fuera del aula y la evaluación. La realidad de la operacionalización de dicho proyecto en el entramado educativo, mediado por las subjetividades, acciones e inacciones de tomadores de decisión en el nivel político, mandos medios y docentes, así como la resistencia, consciente o inconsciente, de docentes, estudiantes y sus familias favorecen la creación de un currículum real diferente al diseñado o esperado. Todo esto, en el marco de la interacción de los sujetos del sistema educativo en la globalización y la educación alterna, construida en medios de comunicación y redes sociales.

\subsection{Currículum como producto}

El currículum como proceso genera un producto curricular, es decir; significados, competencias, interacciones y sensibilidades en los estudiantes, profesores y los centros educativos en general. Esto es la manifestación tangible de una selección y organización de contenidos construida partiendo de la acción e inacción política y técnica de una determinada comisión o grupo de funcionarios. Ellos sustentan, consciente o inconscientemente, sus acciones en fundamentos (disciplinares, psicológicos, filosóficos, sociológicos, antropológicos, económicos, culturales, históricos y políticos), intereses y subjetividades, para establecer un programa oficial de conocimientos, destrezas, habilidades, actitudes y sensibilidades por enseñar y a aprender-desaprender, el cual será supervisado-controlado por el gremio y mediado por creadores de material didáctico (libros, videos y otros), docentes y estudiantes.

Por tanto, la comprensión del currículum desde la complejidad de actores, componentes e interacciones participantes en su diseño e implementación conlleva superar la visión tradicional del producto curricular centrado en el dominio de contenidos por parte del estudiante, para dar paso a una evaluación de lo tangible e intangible sucedida en 
actores (estudiante-profesor), aula, centro educativo, familia, comunidad y sociedad en general.

\subsection{El currículum procesado}

El currículum procesado es la transformación del programa de estudio en contenidos para enseñar o aprender. Se centra en la contextualización curricular y las actividades didácticas-evaluativas implementadas en el aula. Analizado metafóricamente, este currículum puede ser de procesamiento artesanal, cuando es realizado por el docente quien crea los materiales didácticos, o un currículum de procesamiento industrial, es decir, cuando es elaborado por empresas especializadas, como el caso de las editoriales.

En el procesamiento industrial, el currículum oficial es alterado con hormonas para el crecimiento de los contenidos (se priorizan unos sobre otros), se fractura por partes para la venta o se convierte en transgénico, lo que quiere decir un currículum alterado en sus componentes básicos (conocimientos, habilidades, destrezas, actitudes y sensibilidades), mediante la inserción de elementos externos (comentarios, fotografías, colores y otros elementos del material didáctico). De este modo, se genera un contenido similar al oficial, pero con variantes en su $A D N$ que le convierten en sustancialmente diferente.

\subsection{Currículum como práctica educativa}

El currículum es un producto cultural y pedagógico que sistematiza las visiones, intereses y resistencias de tomadores de decisión, grupos de interés (partidos políticos, universidades, editoriales, grupos temáticos y otros), colectivos de presión (sindicatos, cámaras patronales, organizaciones religiosas y de la sociedad civil) y, en mucho menor medida, del docente y del estudiante. Estas visiones son plasmadas en hechos tangibles (infraestructura, programas de estudio, asignaturas, organización administrativa, tiempos pedagógicos y otros) y elementos intangibles (vinculados al currículum oculto propuesto por Torres, 1998) que interactúan con el contexto, para establecer limitantes y posibilidades a la acción del educador como mediador y del estudiante como agente activo del proceso de aprendizaje. 
Por tanto, el currículum es una práctica educativa cuando es insertado en la dinámica de la institución rectora y en la vivencia cotidiana de los centros educativos y de las aulas.

\subsection{Currículum como vivencia educativa}

El currículum como vivencia educativa se da en el momento de la contextualización curricular, es decir, la transposición del diseño curricular a la realidad del aula, mediado por la planificación del docente (planeamiento didáctico o de contextualización curricular) y la acción pasiva-activa del estudiante. Ambos actores asumen, reproducen, transforman e implementan de diferentes formas el currículo, según sea la formación y las subjetividades del agente educativo (mando medio, administrativo, docente, estudiante y familia), de las condiciones pedagógicas, del contexto y del momento histórico

En este punto, el currículum adquiere significados, interpretaciones y acciones, desde la acción de estudiantes y docentes quienes pueden producir, transformar o rebelarse ante los contenidos, habilidades, destrezas, actitudes y sensibilidades impuestas en el programa de estudio. Por tanto, se puede definir como el currículum construido en la interacción de los tres pilares del sistema educativo: estudiante, docente y contenido/competencia.

\subsection{El currículum inesperado}

El currículum inesperado es aquello que sucede como producto de la vivencia educativa, pero que no fue planificado ni será evaluado explícitamente. Las implicaciones del contenido en la vida del estudiante, sus pensamientos, creencias, valoraciones, sentimientos, sensibilidades, destrezas y actitudes, en su vida cotidiana, la convivencia y su proyecto de vida, son parte del currículum inesperado, el cual puede girar desde el olvido total de lo aprendido hasta la oposición (resistencia) consciente y sistemática al contenido, al sistema educativo y hasta al mismo sistema social que legitima la estructura educativa.

\subsection{El currículum reducido o formal}

Esta interpretación es la concepción clásica y dominante, según la cual el currículo se reduce a un plan de estudios o programas de cursos, descontextualizado de su origen e implicaciones, priorizando la 
técnica (selección de cursos, contenidos y actividades) y estableciendo los resultados desde la acción individual del estudiante.

El currículum reducido o formal representa una limitante para el análisis curricular, por la supuesta neutralidad técnica y una amenaza, al representar los procesos curriculares con una tenue línea divisoria, con áreas como la didáctica, evaluación educativa y administración.

A partir de las anteriores definiciones, resulta necesario replantear el papel del curriculista como la persona líder de un proyecto de educación o formación que plasma la interacción de fundamentos, aspiraciones pedagógicas, intereses implícitos u ocultos, realidades pedagógicas (centro educativo, comunidad y sistema) y vivencia educativa, en un documento orientador denominado estructura curricular que sistematiza la experiencia del diseño curricular.

En este contexto, el curriculista es una persona crítica para ver y comprender más allá de los elementos técnicos del currículum; negociadora entre intereses, grupos y personas; tomadora de decisiones; flexible a la hora de interactuar frente a la complejidad e incertidumbre durante el diseño; capaz de evaluar y analizar procesos, intereses, proyecciones de realidad, interacciones, significados, relevancias y pertinencia de elementos teóricos, pedagógicos, contextuales; consciente de lo determinante de la vivencia educativa y del currículum inesperado.

Estas características se plasmarán en un diseño curricular, es decir, el mecanismo mediante el cual la persona curriculista logra sistematizar las cualidades de un procedimiento de formación, manifiesto en un plan de estudios o su mejora, a partir de la construcción del currículum como área del conocimiento, proceso, producto, procesado, práctica educativa, vivencia educativa, inesperado y reducido o formal; todo lo cual se sistematiza parcial o totalmente en la estructura curricular.

Finalmente, la estructura curricular se conceptualiza como un contrato curricular; lo cual significa el documento formal de un plan de estudio que integra un análisis de viabilidad, justificación, fundamentación teórica (disciplinar y pedagógica), misión, visión, objeto de estudio, perfiles de ingreso y salida, objetivos generales y específicos (o propósitos, en caso de competencias), malla curricular, áreas, ejes, distribución de cursos, grado académico, población meta, metas del plan y, finalmente, el programa de los cursos o asignaturas. Esta concepción implica que la estructura curricular es solo un reflejo parcial del currículum, en el que se establece una serie de mínimos necesarios para la 
calidad educativa, pero que debe ser flexible para permitir la constante innovación y evolución de la propuesta pedagógica, tanto desde la formalidad hasta la vivencia del currículum en las aulas.

\section{Conclusiones}

Lo expuesto en el presente ensayo permite visibilizar la diversidad de posibles concepciones del currículum, a partir de paradigmas que los diversos autores tienen sobre la educación, la sociedad, la cultura y otros ejes estructurantes de sus análisis. De este modo, la categorización de los enfoques curriculares con base en las categorías técnica, práctica y crítica permite establecer un contexto de acercamiento a la definición de lo curricular, desde premisas básicas en su construcción.

Adicionalmente, fundamentadas en una posición ecléctica de los enfoques práctico y crítico, así como en delimitaciones contextuales para el caso costarricense, se ha elaborado las concepciones de currículum vinculado al área del conocimiento, currículum como proceso, currículum como producto, currículum procesado, currículum como práctica educativa, currículum como vivencia educativa y currículum inesperado. Estas nociones aportan elementos para la reflexión-acción del currículum costarricense desde su uso y su práctica, permitiendo establecer una diferenciación de conceptos, según sea el hecho por analizar o realizar con (o en) el currículum, pero con elementos, implícitos y explícitos, comunes.

A corto plazo, resulta necesaria la ampliación de las reflexiones sobre el currículum en Costa Rica, con el objetivo de construir concepciones, características y delimitaciones comunes para los encargados de diseñar, investigar e implementar dicho currículum. Igualmente, se requiere un marco para la reflexión-acción sobre las posibilidades de mejora y transformación del mismo currículum en las circunstancias de nuestra sociedad, al iniciar el siglo XXI.

\section{Referencias}

Apple, M. (1996a). El conocimiento oficial. Barcelona: Paidós.

Apple, M. (1996b). Política Cultural y Educación. Barcelona: Paidós. Apple, M. (1997). Educación y poder. Barcelona: Paidós.

Bolaños, G. y Molina, Z. (2007). Introducción al currículo. Costa Rica: EUNED.

Casarini, M. (2005). Teoría y Diseño Curricular. México: Trillas. 
Clemente, M. (2010). Diseñar el currículum prever y representar la acción. En J. Gimeno (comp.), Saberes e incertidumbres sobre el currículum (pp. 269-293). Madrid: Morata.

Escudero, J. et al. (2000). Diseño, desarrollo e innovación del currículum. Madrid: Síntesis Educativa.

Freire, P. (1997). Educación y participación comunitaria. En M. Castells, R. Flecha, P. Freire, H. Giroux, D. Macedo y P. Willis (eds.), Nuevas perspectivas críticas en educación (pp. 83-96). Barcelona: Paidós.

Freire, P. (1990). La naturaleza política de la educación. Cultura, poder y liberación. Barcelona: Paidós.

Freire, P. (1984). Pedagogía del Oprimido. Madrid: Siglo XXI.

Garbanzo, G. (2001). La administración curricular en la educación superior caso de la Universidad de Costa Rica. Revista Educación 25(2), 25-39.

Gimeno, J. (2010a). ¿Qué significa el currículum? En J. Gimeno (comp.), Saberes e incertidumbres sobre el currículum (pp. 2144). Madrid: Morata.

Gimeno, J. (2010b). El currículum en la sociedad de la información y del conocimiento. En J. Gimeno (comp.), Saberes e incertidumbres sobre el curriculum (pp. 180-202). Madrid: Morata.

Gimeno, J. (2001). El currículum: una reflexión sobre la práctica. España: Morata.

Gimeno, J. (1997). La pedagogía por objetivos: Obsesión por la eficiencia. España: Morata.

Giroux, H. (1997). Cruzando los límites. Trabajadores culturales y políticas educativas. España: Paidós.

González, M. (2010). Los colectivos depauperados repolitizan los currícula. En J. Gimeno (comp.), Saberes e incertidumbres sobre el currículum (pp. 128-148). Madrid: Morata.

González, V. (2018). La coherencia curricular en la Educación Superior: algunas reflexiones. Revista Educación, 42(2), 1-17.

Grundy, S. (1998). Producto o praxis del currículum. Madrid: Morata.

Guirtz, S. y Palamidessi, M. (1998). El ABC de la tarea docente: currículum y enseñanza. Argentina: AIQUE. Recuperado de http:// www.atencapital.org.ar/sites/default/files/Ensenanza.pdf

Jiménez, L. (2008). Enfoque curricular centrado en la persona. Revista Educación, 32(1), 63-76. 
Kemmis, S. (1998). El currículum: más allá de la teoría de la reproducción. Madrid: Morata.

Lundgren, U. (1992). Teoría del currículum y escolarización. Madrid: Morata.

McLaren, P. (1997). Pedagogía crítica y cultura depredadora. Barcelona: Paidós.

Magendzo, A. (2008). Dilemas del currículum y la pedagogía: analizando la Reforma Curricular desde una perspectiva crítica. Santiago: LOM.

Mora, A. (2001). Los contenidos curriculares del plan de estudios: Una propuesta para su organización y estructura. Revista Educación, 25(2), 147-156.

Mora, A. y Herrera, M. (1998). Lineamientos para elaborar el perfil académico profesional. San José: Instituto de Investigaciones para el Mejoramiento de la Educación Costarricense.

Rodríguez, C. (2013). El potencial curricular de los libros de texto para generar experiencias de aprendizaje. Revista Educación, 37(1), 119-129.

Stenhouse, L. (2003). Investigación y desarrollo del currículum. Madrid: Morata.

Taba, H. (1974). Elaboración del Currículo. Teoría y Práctica. Buenos Aires: Troquel.

Torres, J. (2010). Currículum, justicia e inclusión. En J. Gimeno (comp.), Saberes e incertidumbres sobre el currículum (pp. 84102). Madrid: Morata.

Torres, J. (1998). El currículum oculto (sexta edición). Madrid: Morata.

Tyler, R. (1973). Principios básicos del currículum. Buenos Aires: Troquel.

Zabalza, M. (2004). Diseño y desarrollo curricular. Madrid: Narcea. 
